\title{
VAE-Sim: A Novel Molecular Similarity Measure Based on a Variational Autoencoder
}

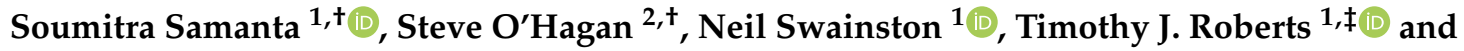 \\ Douglas B. Kell 1,3,*iD \\ 1 Department of Biochemistry and Systems Biology, Institute of Systems, Molecular and Integrative Biology, \\ University of Liverpool, Crown St, Liverpool L69 7ZB, UK; soumitra.samanta@liverpool.ac.uk (S.S.); \\ neil.swainston@liverpool.ac.uk (N.S.); timothy-roberts@outlook.com (T.J.R.) \\ 2 Department of Chemistry, The Manchester Institute of Biotechnology, The University of Manchester, \\ 131 Princess St, Manchester M1 7DN, UK; sohagan@manchester.ac.uk \\ 3 Novo Nordisk Foundation Centre for Biosustainability, Technical University of Denmark, Building 220, \\ Kemitorvet, 2800 Kgs Lyngby, Denmark \\ * Correspondence: dbk@liv.ac.uk \\ + These authors contributed equally to this work. \\ $\ddagger$ Present address: University College London Hospital NHS Foundation Trust, 250 Euston Road, \\ London NW1 2PB, UK.
}

Academic Editor: Derek J. McPhee

Received: 26 June 2020; Accepted: 28 July 2020; Published: 29 July 2020

\begin{abstract}
Molecular similarity is an elusive but core "unsupervised" cheminformatics concept, yet different "fingerprint" encodings of molecular structures return very different similarity values, even when using the same similarity metric. Each encoding may be of value when applied to other problems with objective or target functions, implying that a priori none are "better" than the others, nor than encoding-free metrics such as maximum common substructure (MCSS). We here introduce a novel approach to molecular similarity, in the form of a variational autoencoder (VAE). This learns the joint distribution $\mathrm{p}(\mathrm{z} \mid \mathrm{x})$ where $\mathrm{z}$ is a latent vector and $\mathrm{x}$ are the (same) input/output data. It takes the form of a "bowtie"-shaped artificial neural network. In the middle is a "bottleneck layer" or latent vector in which inputs are transformed into, and represented as, a vector of numbers (encoding), with a reverse process (decoding) seeking to return the SMILES string that was the input. We train a VAE on over six million druglike molecules and natural products (including over one million in the final holdout set). The VAE vector distances provide a rapid and novel metric for molecular similarity that is both easily and rapidly calculated. We describe the method and its application to a typical similarity problem in cheminformatics.
\end{abstract}

Keywords: cheminformatics; molecular similarity; deep learning; variational autoencoder; SMILES

\section{Introduction}

The concept of molecular similarity lies at the core of cheminformatics [1-3]. It implies that molecules of "similar" structure tend to have similar properties. Thus, a typical question can be formulated as follows: "given a molecule of interest $\mathrm{M}$, possibly showing some kind of chemical activity, find me the nearest 50 molecules from a potentially huge online collection to purchase that are most similar to $\mathrm{M}$ so I can assess their behaviour in a relevant quantitative-structure-activity (QSAR) analysis".

The most common strategies for assessing molecular similarity involve encoding the molecule as a vector of numbers, such that the vectors encoding two molecules may be compared according to their Euclidean or other distance. In the case of binary strings, the Jaccard or Tanimoto similarity 
(TS) is commonly used [4] as a metric (between zero and one). One means for obtaining such a vector for a molecule is to calculate from the structure (or measure) various properties of the molecule ("descriptors" [5-7]), such as clogP or total polar surface area, and then to concatenate them. However, a more common strategy for obtaining the encoding vector of numbers is simply to use structural features directly and to encode them as so-called molecular fingerprints [8-17]. Well-known examples include MACCS [18], atom pairs [19], torsion [20], extended connectivity [21], functional class [22], circular [23], and so on. The similarities so encoded can also then be compared as their Jaccard or Tanimoto similarities. Sometimes a "difference" or "distance" is discussed and formulated as 1-TS (a true metric). An excellent and widely used framework for performing all of this is RDKit (Pathon v3.6.8) (www.rdkit.org/) [24], that presently contains nine methods for producing molecular fingerprints.

The problem comes from the fact that the "most similar" molecules to a target molecule often differ wildly both as judged by their structures observable by eye and quantitatively in terms of the value of the TS of the different fingerprints [25]. As a very small and simple dataset, we take the set of molecules observed by Dickens and colleagues [26] to inhibit the transporter-mediated uptake of the second-generation atypical antipsychotic drug clozapine. These are olanzapine, chlorpromazine, quetiapine, prazosin, lamotrigine, indatraline, verapamil and rhein. Of the FDA-approved drugs, we assessed the top 50 drugs in terms of their structural similarity to clozapine using the nine RDKit encodings, with the results shown in Table 1 and Figure 1 (see also Supplementary Figure S1). Only the first four of these are even within the top 50 for any encoding, and only olanzapine appears for each of them. By contrast, the most potent inhibitor is prazosin (which is not even wholly of the same drug class, being both a treatment for anxiety and a high-blood-pressure-lowering agent); however, it appears in the top 50 in only one encoding (torsion) and then with a Tanimoto similarity of just 0.37. That said, visual inspection of their "Kekularised" structures does show a substantial common substructure between prazosin and clozapine (marked in Figure 1). It is clear that the similarities, as judged by standard fingerprint encodings, are highly variable, and are prone to both false negatives and false positives when it comes to attacking the question as set down above. What we need is a different kind of strategy.

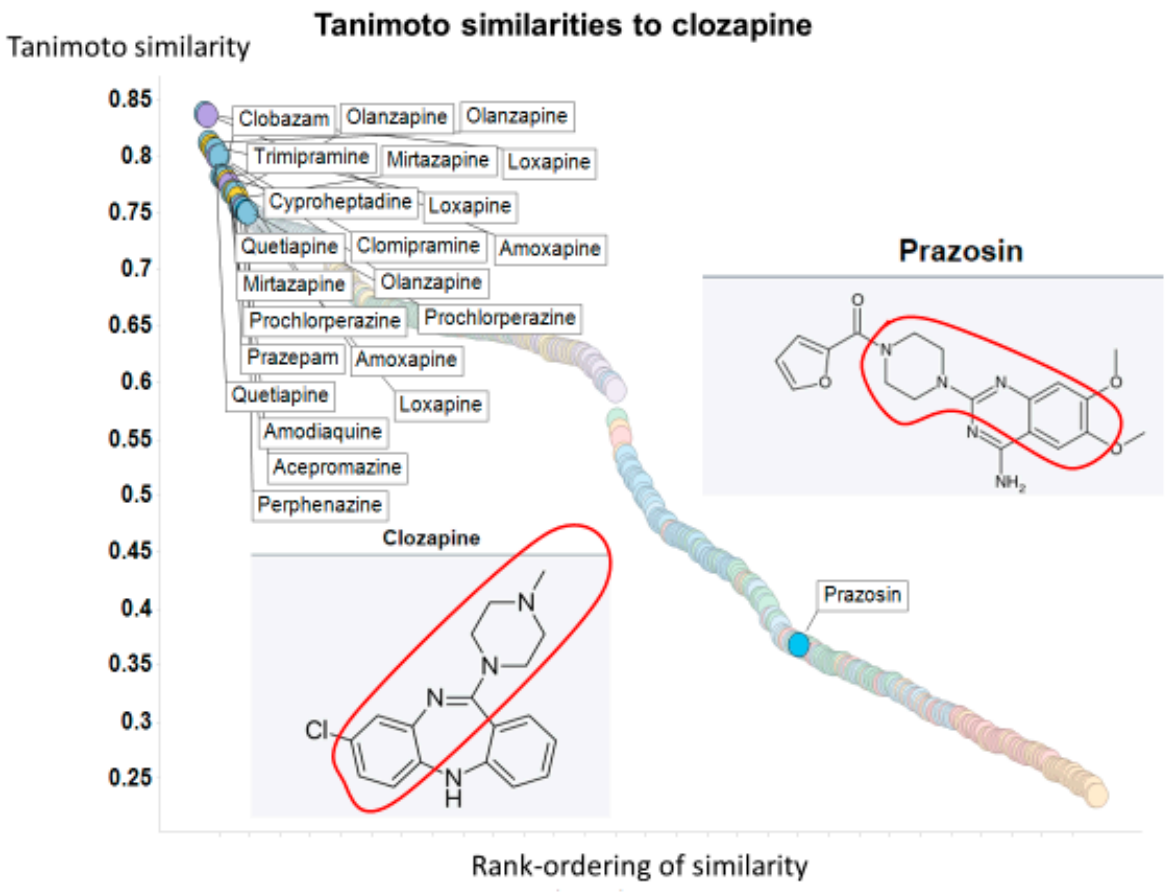

Figure 1. Tanimoto similarities of various molecules to clozapine using the Torsion encoding from RDKit. 
Table 1. Tanimoto similarity to clozapine using nine different RDKit encodings and their ability to inhibit clozapine transport (data extracted from [26]). A shaded cell means that the molecule was not judged to be in the "top 50 " using that encoding.

\begin{tabular}{|c|c|c|c|c|c|c|c|c|c|c|}
\hline Drug & $\begin{array}{c}\text { \% Inhiclozapine } \\
\text { Uptake }\end{array}$ & $\begin{array}{l}\text { TS Atom } \\
\text { Pair }\end{array}$ & TS Avalon & $\begin{array}{l}\text { TS Feat } \\
\text { Morgan }\end{array}$ & $\begin{array}{c}\text { TS } \\
\text { Layered }\end{array}$ & $\begin{array}{c}\text { TS } \\
\text { MACCS }\end{array}$ & $\begin{array}{c}\text { TS } \\
\text { Morgan }\end{array}$ & TS Pattern & TS RDKit & $\begin{array}{c}\text { TS } \\
\text { Torsion }\end{array}$ \\
\hline Olanzapine & 41 & 0.68 & 0.47 & 0.55 & 0.77 & 0.8 & 0.53 & 0.81 & 0.74 & 0.66 \\
\hline Chlorpromazine & 75 & 0.53 & - & 0.35 & - & 0.66 & 0.3 & 0.74 & - & 0.33 \\
\hline Quetiapine & 65 & 0.51 & 0.57 & 0.42 & 0.78 & - & 0.35 & 0.8 & - & 0.48 \\
\hline Prazosin & 94 & - & - & - & - & - & - & - & - & 0.37 \\
\hline Lamotrigine & 26 & - & - & - & - & - & - & - & - & - \\
\hline Indatraline & 35 & - & - & - & - & - & - & - & - & - \\
\hline Veraapamil & 83 & - & - & - & - & - & - & - & - & - \\
\hline Rhein & 39 & - & - & - & - & - & - & - & - & - \\
\hline
\end{tabular}


The typical structure of a QSAR type of problem is provided in Figure 2A, where a series of molecules represented as SMILES strings [27] are encoded as molecular fingerprints and used to learn a nonlinear mapping to produce an output in the form of a classification or regression estimation. The architecture of this is implicitly in the form of a multilayer perceptron (a classical neural network [28-31]), in which weights are modified ("trained") to provide a mapping from input SMILES to a numerical output. Our fundamental problem stems from the fact that these types of encoding are one-way: the SMILES string can generate the molecular fingerprint but the molecular fingerprint cannot generate the SMILES. Put another way, it is the transition from a world of discrete objects (here molecules) with categorical representations (here SMILES strings) to one of a continuous representation (vectors of weights) that is seemingly irreversible in this representation. One key element is the means by which we can go from a non-numerical representation (such as SMILES or similar [32]) to a numerical representation or "embedding" (this, as we shall see, is typically constituted by vectors of numbers in the nodes and weights of multilayer neural networks) [33-38]. Deep learning has also been used for the encoding step of 2D chemical structures [39,40].

More recently, it was recognised that various kinds of architectures could, in fact, permit the reversal of this numerical encoding so as to return a molecule (or its SMILES string encoding a unique structure). These are known as generative methods [41-55], and at heart their aim to generate a suitable and computationally useful representation [56] of the input data. It is common (but cf. [57,58]) to contrast two main flavours: generative adversarial networks [59-66] and (especially variational) autoencoders (VAEs) [41,42,67-76]. We focus here on the latter, illustrated in Figure 2B.

VAEs are latent-variable generative models that define a joint density $\mathrm{p}_{\theta}(\mathrm{x}, \mathrm{z})$ between some observed data $\mathrm{x} \in \mathrm{R}^{\mathrm{dx}}$ and unobserved or latent variables $\mathrm{z} \in \mathrm{R}^{\mathrm{dz}}$ [77], given some model parameters $\theta$. They use a variational posterior (also referred to as an encoder), $\mathrm{q}_{\varphi}(\mathrm{z} \mid \mathrm{x})$, to construct the latent variables with variational parameters $\varphi$, and a combination of $\mathrm{p}(\mathrm{z})$ and $\mathrm{p}(\mathrm{x} \mid \mathrm{z})$ to create a decoder that has the opposite effect. Learning the posterior directly is computationally intractable, so the generic deep learning strategy is to train a neural network to approximate it. The original "error" backpropagated was based on the Kullback-Leibler (KL) divergence between the desired (log likelihood reconstruction error) and the predicted output distributions [67]. A very great many variants of both architectures and divergence metrics have been proposed since then (not all discernibly better [78]), and it is a very active field (e.g., [63,64,79-83]). Since tuning is necessarily domain-specific [84], and most work is in the processing of images and natural languages rather than in molecules, we merely mention a couple, such as transformers (e.g., $[85,86])$ and others (e.g., $[87,88])$. Crucial to such autoencoders (that can also be used for data visualisation [89]) is the concept of a bottleneck layer, that as a series of nodes of lower dimensionality than its predecessors or successors, serves to extract or represent [56] the crucial features of the input molecules that are nonetheless sufficient to admit their reconstruction. Indeed, such strategies are sometimes referred to as representational learning.

A higher-level version of the above might state that a good variational autoencoder will project a set of discrete molecules into a continuous latent space represented for any given molecule by the vector representing the values of the outputs of the nodes in the bottleneck layer when it (or its SMILES representation) is applied to the encoder as an input. As with the commonest neural net training system (but cf. [90-94]), we use backpropagation to update the network so as to minimise the difference between the predicted and the desired output, subject to any other constraints that we may apply. We also recognise the importance of various forms of regularisation, that are all designed to prevent overfitting [49,95-98].

Because the outputs of the nodes in the bottleneck layer both (i) encode the molecule of interest and (ii) effectively represent where molecules are in the chemical space on which they have been trained, a simple metric of similarity between two molecules is clearly the Euclidean or other comparable distance (e.g., cosine distance) between these vectors. This thus provides for a novel type of similarity encoding, that in a sense relates the whole chemical space on which the system has been trained and 
that we suspect may be of general utility. We might refer to this encoding as the "essence of molecules" (EM) encoding, but here, we refer to it as VAE-Sim.
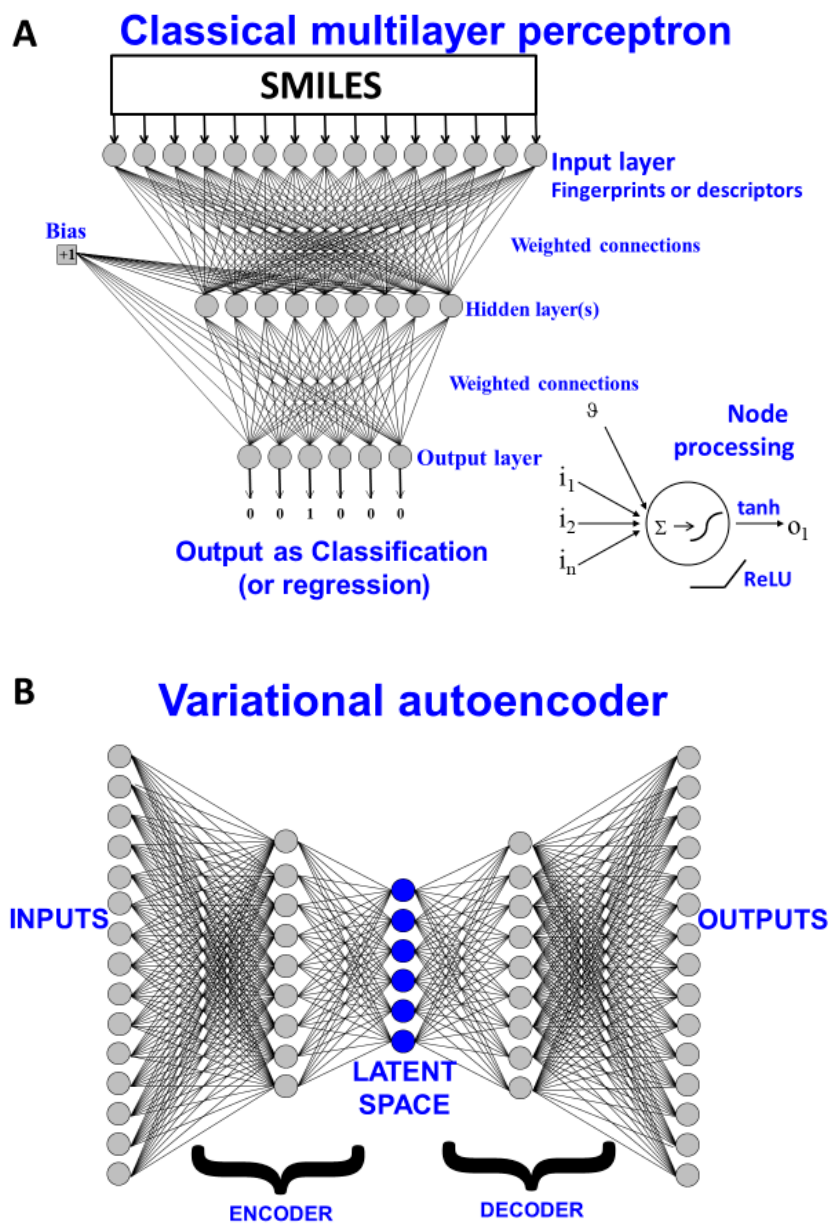

\section{C \\ Overall architecture of the present variational autoencoder for molecule reconstruction}

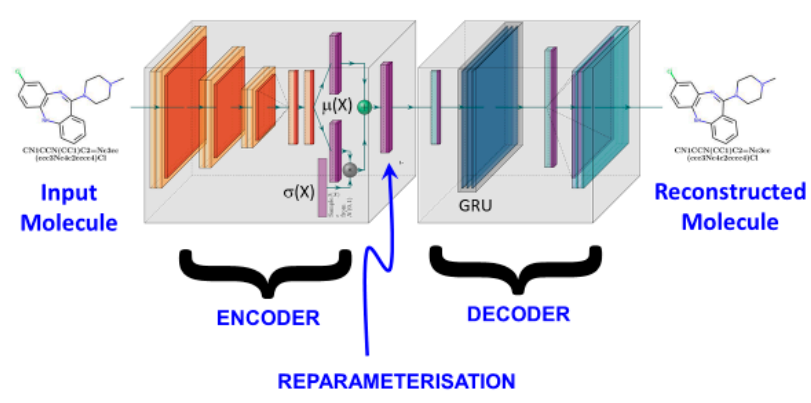

Figure 2. Two kinds of neural architecture. (A) A classical multilayer perceptron representing a supervised learning system in which molecules encoded as SMILES strings can be used as paired inputs with outputs of interest (whether a classification or a regression). The trained model may then be interrogated with further molecules and the output ascertained. (B) A variational autoencoder, is a supervised means of fitting distributions of discrete models in a way that reconstructs them via a vector in a latent space. (C) The variational autoencoder (VAE) architecture used in the present work. 
Thus, the purpose of the present article is to describe our own implementation of a simple VAE and its use in molecular similarity measurements as applied, in particular, to the set of drugs, metabolites and natural products that we have been using previously [25,99-104] as our benchmark for similarity metrics. A preprint was deposited at bioRxiv [105].

\section{Results}

Autoencoders that use SMILES as inputs can return three kinds of outputs: (i) the correct SMILES output mirroring the input and/or translating into the input molecular structure (referred to as "perfect"), (ii) an incorrect output of a molecule different from the input but that is still legal SMILES (hence will return a valid molecule), referred to as "good", and (iii) a molecule that is simply not legal SMILES. In practice, our VAE after training returned more than 95\% valid SMILES in the test (holdout) set, so those that were invalid could simply be filtered out without significant loss of performance.

Specifically, we partitioned the dataset into $50 \%$ for training $(3,101,207$ samples $), 20 \%$ for validation $(1,240,483$ samples) and $30 \%$ for testing (1,860,725 samples). The results for our VAE model for ZINC molecule reconstruction are as follows (Table 2):

Table 2. Data partitioning of training, validation and test sets, and their generalization.

\begin{tabular}{cccc}
\hline Data Partition & Total Samples & Valid Reconstructed Samples & Accuracy \\
\hline Train & $3,101,207$ & $2,964,749$ & 95.60 \\
\hline Validation & $1,240,483$ & $1,170,827$ & 94.38 \\
\hline Test & $1,860,725$ & $1,757,079$ & 94.42 \\
\hline
\end{tabular}

Following training, each molecule (SMILES) could be associated with a normalised vector of 100 dimensions, and the Euclidean distance between them could be calculated.

$$
\begin{aligned}
& E-\operatorname{Sim}(x, y)=1 /\left(1+\sqrt{\sum_{i=1}^{100}\left(x_{i}-y_{i}\right)^{2}}\right) ; x, y \in R^{100} \\
& E U-\operatorname{Sim}(x, y)=1 /\left(1+\sqrt{\sum_{i=1}^{2}\left(x_{i}-y_{i}\right)^{2}}\right) ; x, y \in R^{2}
\end{aligned}
$$

As previously described [25], we compared the similarities between all drugs and all metabolites using the datasets made available in [99]. We here focus on just the MACCS and Patterned encodings of RDKit, and compare them with the normalised Euclidean distances according to the latent vector obtained from the VAE. As before, we rank ordered each drug in terms of its closest similarity to any metabolite. First, Figure 3A (reading from right to left) shows the Tanimoto similarities for the Patterned and MACCS fingerprints, as well as the VAE-Sim values as judged by two metrics. The first, labelled E-Sim Equation (1), is the Euclidean similarity, based on the raw 100-dimensional hidden vectors, while the second, EU-Sim Equation (2), used the Uniform Manifold Approximation and Projection (UMAP) dimension reduction algorithm [106,107] based on the first two UMAP dimensions was used for purposes of visualisation; clearly, as with other encodings, they do not at all follow the same course, and one that may be modified according to the similarity measure used. Figure 3B,C show the "all-vs.-all" heatmaps for two of the encodings, indicating again that the VAE-Sim encoding falls away considerably more quickly, i.e., that similarities are judged in a certain sense more "locally".

Figure 4A shows the Patterned similarity for the "most similar" metabolite for each drug (using TS) compared to that for VAE-Sim (using Euclidean distance), while Figure 4B shows the same for the MACCS encoding. These, again, illustrate how the new encoding provides a quite different readout from the standard fingerprint encodings. 

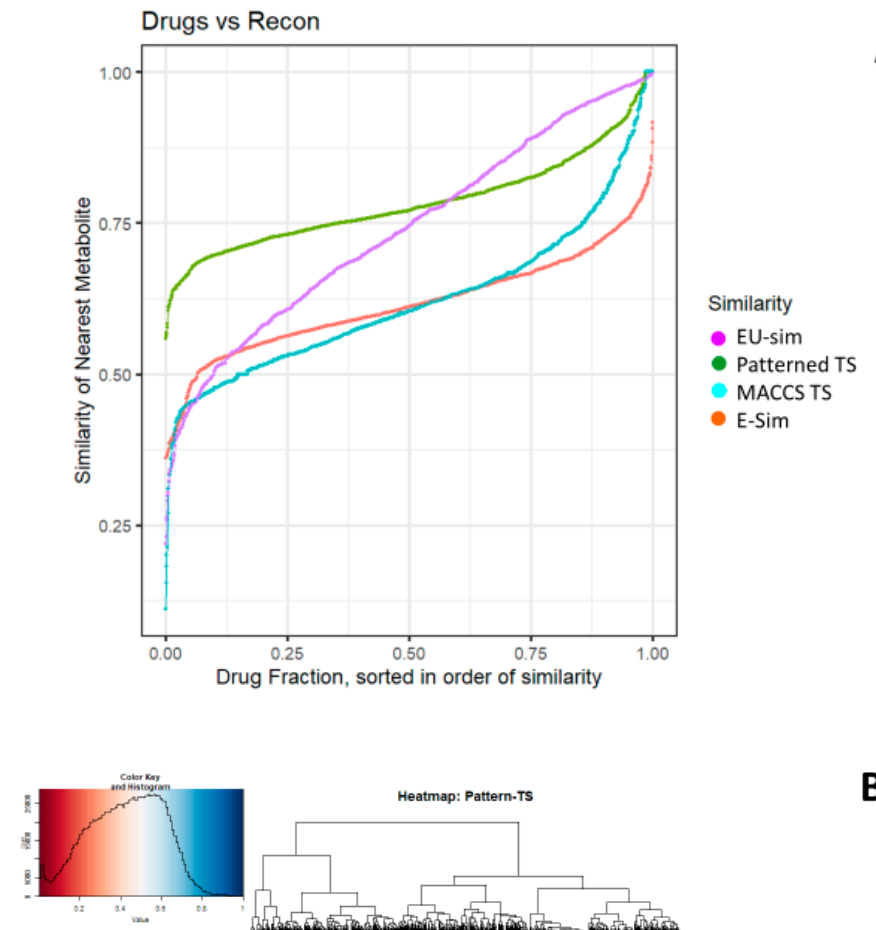

A

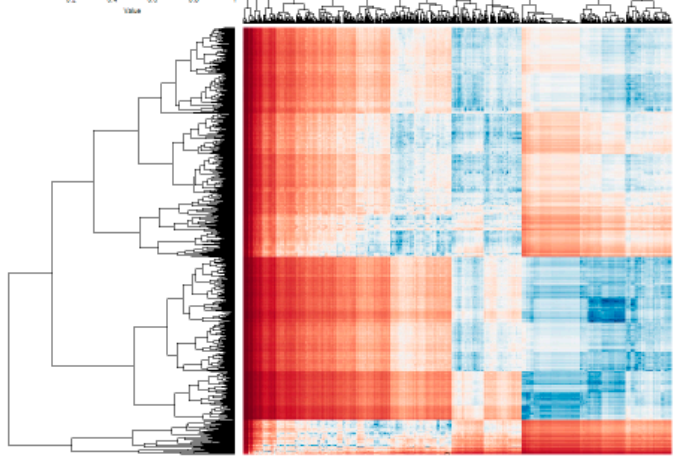

Drugs

Recon2

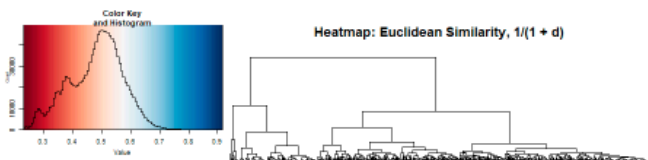

C

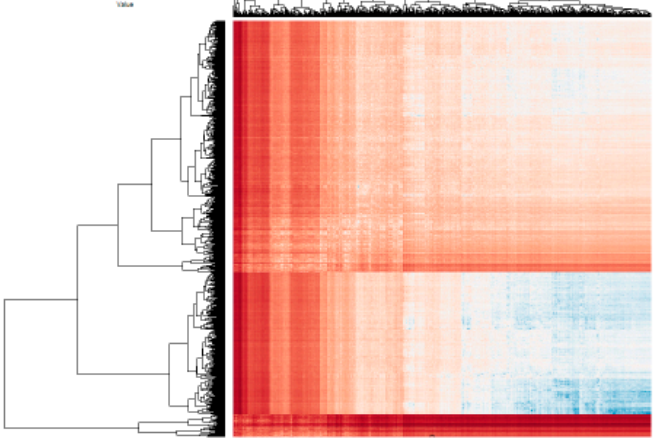

B

\section{Drugs}

Drugs

Figure 3. Cont. 


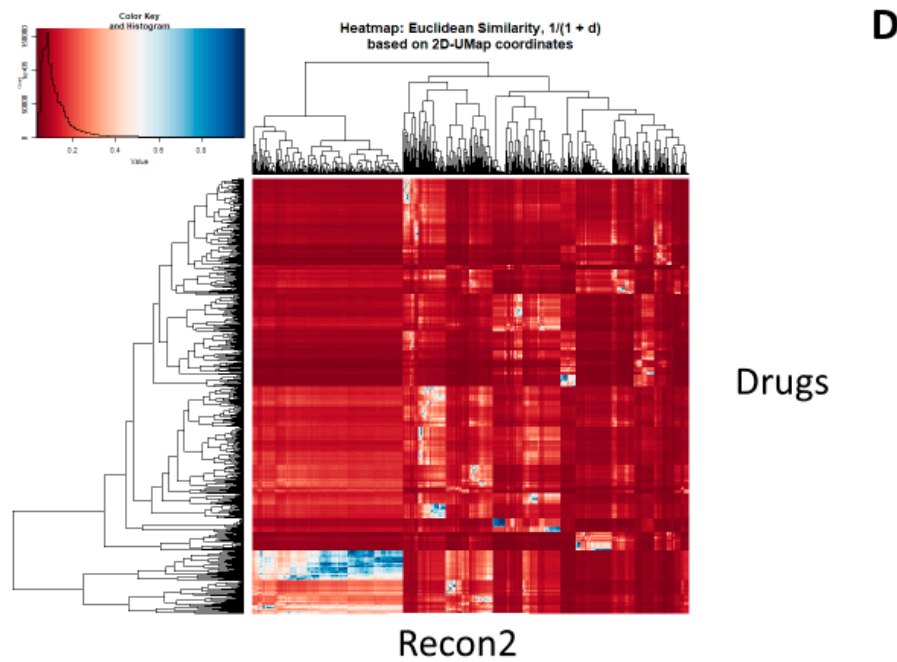

Figure 3. Top similarities between drugs and metabolites as judged by a fingerprint encoding (RDKit patterned) and our new VAE-Sim metric. (A) Rank ordering. (B) Heatmap for Tanimoto similarities using RDKit patterned encoding. (C) Heatmap of Euclidean similarities E-Sim (Equation (1)) for VAE-Sim in the 100-dimensional latent vector). (D) Heatmap of Euclidean similarities EU-Sim (Equation (2)) for VAE-Sim in 2-dimensional uniform manifold approximation and projection (UMAP) space.

\section{A}

\section{Patterned vs VAE-Sim}

Patterned TS

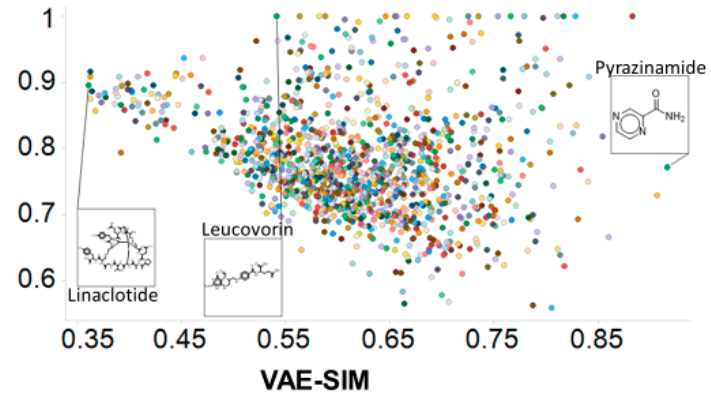

\section{B MACCS vs VAE-Sim}

MACCS TS

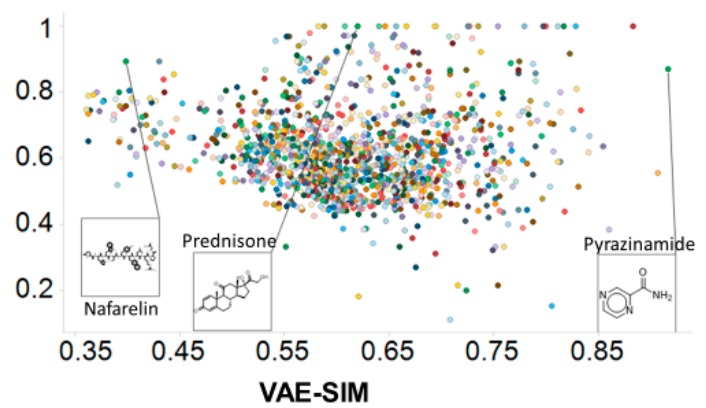

Figure 4. Comparison of similarities between two RDKit fingerprint methods and VAE-Sim Using Tanimoto similarity for fingerprints and Euclidean $\mathrm{d}_{100}$ similarity for VAE-Sim. (A) Patterned encoding. (B) MACCS encoding. 
Finally, we used our new metrics to determine the similarity to clozapine of other drugs. Figure 5 shows the two similarity scores based on VAE-Sim, calculated as in Figure 3. Gratifyingly, and while structural similarity is, in part, in the eye of the beholder, a variety of structurally and functionally related antipsychotic drugs such as loxapine, mirtazapine and quetiapine were indeed among the most similar to clozapine, while others not previous considered (such as the antihistamines ketotifen and alcaftadine, and the anti-inflammatory COX inhibitor ketorolac) were also suggested as being similar, providing support for the orthogonal utility of the new VAE-Sim metric. However, the rather promiscuous nature of clozapine binding (e.g., [108,109]), along with that of many of the other drugs (e.g., [110-116]), means that this is not the place to delve deeper.
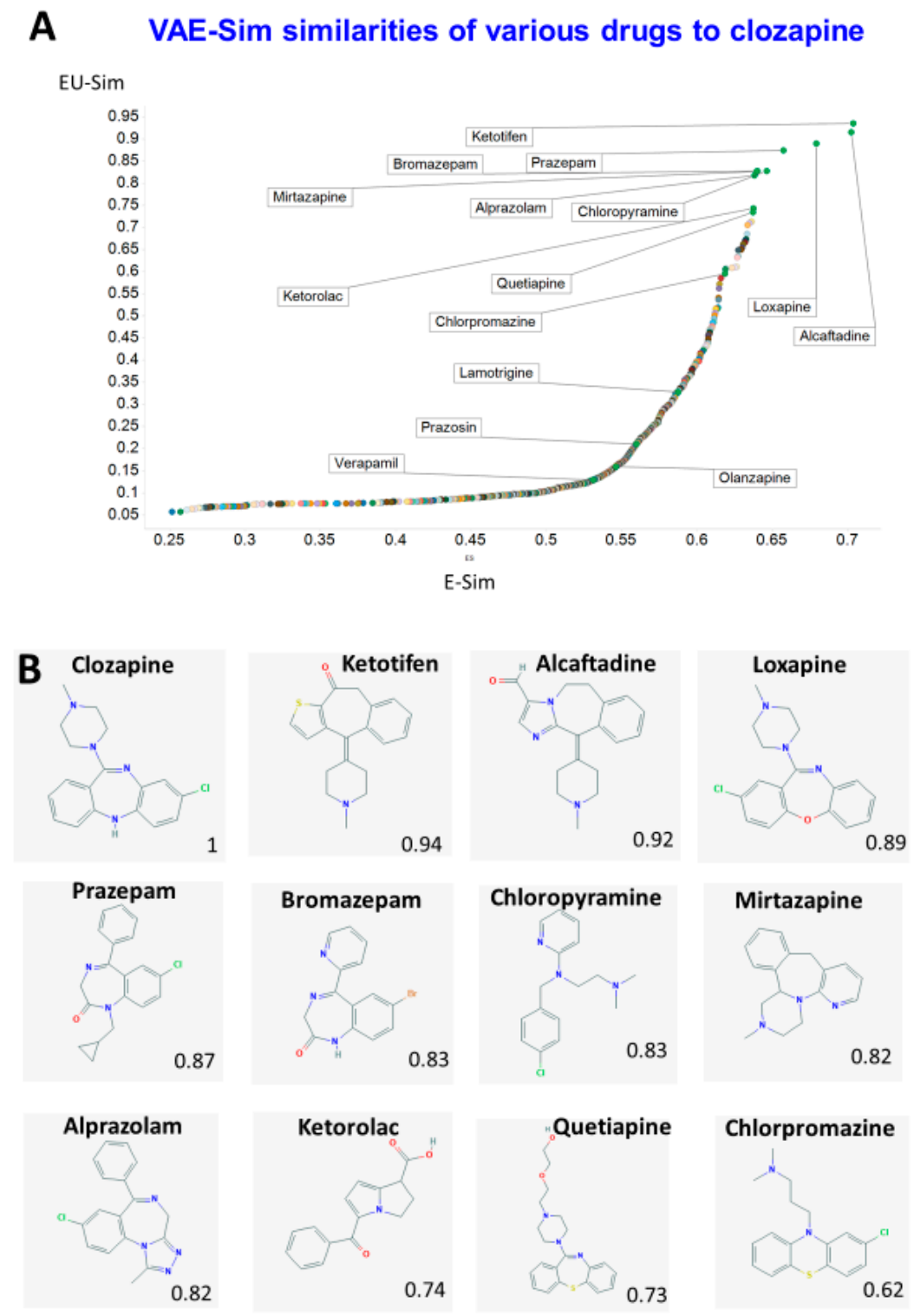

Figure 5. Similarity of drugs to clozapine as judged by the VAE. (A) Rank order of Euclidean similarity in 100 dimensions (E-Sim) or two UMAP dimensions (EU-Sim) as in Figure 3. Some of the "most similar" drugs are labelled, as are some of those in Table 1. (B) Structures of some of the drugs mentioned, together with their Euclidean distances as judged by VAE-Sim. 


\section{Methods}

We considered and tested grammar-based and junction-tree methods such as those used by Kajino [35], that exploited some of the ideas developed by Dai [117], Kusner [118] and by Jin and their colleagues [34]. However, our preferred method as described here used one-hot encoding as set out by Gómez-Bombarelli and colleagues [74]. We varied the number of molecules in the training process from ca 250,000 to over 6 million; the large number of possible hyperparameters would have led to a combinatorial explosion, so exhaustive search was (and is) not possible. The final architecture used here (shown in Figure 2C) required 6 days' training on a 1-GPU machine. It involved a convolutional neural network (CNN) encoder with the following layers (Figure 2C): convolution (1D): size (in-248 = SMILES string length, 40 possible unique SMILES characters, out-9, kernel_size = 9), ReLU, convolution (1D): size (in-9, out-9, kernel_size = 9) ReLU, convolution (1D): size (in-9, out-10, kernel_size $=11)$ ReLU, Linear (fully connected): size $(140$, latent_dims = 100) SeLU, with VAE mean-Linear (fully connected): size(140, latent_dims $=100)$ and variance-Linear (fully connected): size $(140$, latent_dims $=100)$. For the decoder we used a Reparameterization (combined mean and sigma together) such that the output will be the same as the latent dimension (100 in our case), Linear (fully connected): size(latent_dims $=100$, latent_dims $=100$ ) SeLU, RNN-GRU (gated neural unit): size (hidden size $=488$, num_layers $=3$ ), Linear (fully connected): size(in-488 = hidden_gru_size, out-248 = SMILES length) Softmax. For the loss we used binary cross-entropy + KL-divergence. Neither dropout nor pooling were used. The optimiser was ADAM [119], the fixed learning rate 0.0001, parameters were initialised using the "Xavier uniform" scheme [120], and a batch size of 128. This was implemented in Python using the Pytorch library (Python v3.8.5). (https://pytorch.org/). Most of the pre- and post-processing cheminformatics workflows were written in the KNIME environment (see [121]).

For the UMAP settings, we used the default parameter settings implemented in the RAPIDS toolbox (ArcGIS v10.2.6), as detailed here https://docs.rapids.ai/api/cuml/stable/api.html\#umap.

\section{Discussion}

Molecular similarity is at the core of much of cheminformatics (e.g., [3,8,122-125]), but is an elusive concept. Our chief interest typically lies in supervised methods such as QSARs, where we use knowledge of paired structures and activities to form a model that allows us to select new structures with potentially desirable activities. Modern modelling methods such as feedforward artificial neural networks based on multilayer perceptrons are very powerful (and they can in fact fit any nonlinear function-the principle of "universal approximation" [126,127]). Under these circumstances, it is usually possible to learn a QSAR using any of the standard fingerprints. However, what we are focused on here is a purely unsupervised representation of the structures themselves (cf [37] which used substructures), and the question of which of these are the "most similar" to a query molecule of interest. Such unsupervised methods may be taken to include any kinds of unsupervised clustering too (e.g., [128-132]). As with any kind of system of this type, the "closeness" is a function of the weighting of any individual features, and it is perhaps not surprising that the different fingerprint methods give vastly different similarities, even when judged by rank order (e.g., [25] and above). One similarity measure that is independent of any fingerprint encoding is represented by the maximum common substructure (MCSS). However, by definition, the MCSS uses only part of a molecule; it is also computationally demanding $[101,102]$, such that "all-against-all" comparisons such as those presented here are out of the question for large numbers of molecules.

Here, we leveraged a new method that uses only the canonical SMILES encoding of the molecules themselves, leading to its representation as a 100-element vector. Simple Euclidean distances can be used to obtain a metric of similarity that, unlike MCSS, is rapidly calculated for any new molecule, even against the entire set of molecules used in the development of the latent space.

In addition, unlike any of the other methods described, methods such as VAEs are generative: moving around in the latent space and applying the vector so created to the decoder allows for the 
generation of entirely new molecules (e.g., [41-45,48,50,63,65,73,74,133]). This opens up a considerable area of chemical exploration, even in the absence of any knowledge of bioactivities.

What Determines the Extent to Which VAEs can Generate Novel Examples?

The ability of variational autoencoders to generalise is considered to be based on learning a certain "neighbourhood" around each of the training examples [77,134], seen as a manifold of lower dimensionality than the dimensionality of the input space [56]. Put another way, "the reconstruction obtained from an optimal decoder of a VAE is a convex combination of examples in the training data" [135]. On this basis, an effect of training set size on the improvement of generalisation (here defined simply as being able to return an accurate answer from a molecule not in the training set) is to be expected, and our ability to generalise (as judged by test set error) improved as the number of molecules increased up to a few million. However, although we did not explore this, it is possible that our default architecture was simply too large for the smaller number of molecules, as excessive "capacity" can cause a loss of generalisation ability [135]. This, of course, leaves open the details of the size and "closeness" of that neighbourhood, how it varies with the encoding used (our original problem) and what features are used in practice to determine that neighbourhood. The network described here took nearly a week to train on a well-equipped GPU-based machine, and exhaustive analysis of hyperparameters was not possible. Consequently, because an understanding of the importance of local density will vary as a function of the position and nature of the relevant chemical space, we are not going to pursue them here. What is important is (i) that we could indeed learn to navigate these chemical spaces, and (ii) that the VAE approach permits a straightforward and novel estimation of molecular similarity.

Supplementary Materials: The following are available online at http://www.mdpi.com/1420-3049/25/15/3446/s1, Figure S1: Molecules similar to clozapine as judged by molecular fingerprint encodings.

Author Contributions: Conceptualization, all authors; methodology, all authors; software, S.S., S.O., N.S.; resources, D.B.K.; data curation, S.S., S.O., N.S.; writing—original draft preparation, D.B.K.; writing-review and editing, all authors; funding acquisition, D.B.K., T.J.R. All authors have read and agreed to the published version of the manuscript.

Funding: Present funding includes part of the EPSRC project SuSCoRD (EP/S004963/1), partly sponsored by AkzoNobel. DBK is also funded by the Novo Nordisk Foundation (grant NNF10CC1016517). Tim Roberts is funded by the NIHR UCLH Biomedical Research Centre.

Conflicts of Interest: The authors declare that they have no conflicts of interest.

\section{References}

1. Gasteiger, J. Handbook of Chemoinformatics: From Data to Knowledge; Wiley/VCH: Weinheim, Germany, 2003.

2. Leach, A.R.; Gillet, V.J. An Introduction to Chemoinformatics; Springer: Dordrecht, The Netherlands, 2007.

3. Maggiora, G.; Vogt, M.; Stumpfe, D.; Bajorath, J. Molecular similarity in medicinal chemistry. J. Med. Chem. 2014, 57, 3186-3204. [PubMed]

4. Willett, P. Similarity-based data mining in files of two-dimensional chemical structures using fingerprint measures of molecular resemblance. Wires Data Min. Knowl. 2011, 1, 241-251. [CrossRef]

5. Todeschini, R.; Consonni, V. Molecular Descriptors for Cheminformatics; Wiley-VCH: Weinheim, Germany, 2009.

6. Ballabio, D.; Manganaro, A.; Consonni, V.; Mauri, A.; Todeschini, R. Introduction to mole db-On-line molecular descriptors database. Math Comput. Chem. 2009, 62, 199-207.

7. Dehmer, M.; Varmuza, K.; Bonchev, D. Statistical Modelling of Molecular Descriptors in QSAR/QSPR; Wiley-VCH: Weinheim, Germany, 2012.

8. Bender, A.; Glen, R.C. Molecular similarity: A key technique in molecular informatics. Org. Biomol. Chem. 2004, 2, 3204-3218. [CrossRef] [PubMed]

9. Nisius, B.; Bajorath, J. Rendering conventional molecular fingerprints for virtual screening independent of molecular complexity and size effects. ChemMedChem 2010, 5, 859-868. [CrossRef]

10. Owen, J.R.; Nabney, I.T.; Medina-Franco, J.L.; López-Vallejo, F. Visualization of molecular fingerprints. J. Chem. Inf. Model 2011, 51, 1552-1563. [CrossRef] 
11. Riniker, S.; Landrum, G.A. Similarity maps-A visualization strategy for molecular fingerprints and machine-learning methods. J. Cheminform. 2013, 5, 43. [CrossRef]

12. Vogt, M.; Bajorath, J. Bayesian screening for active compounds in high-dimensional chemical spaces combining property descriptors and molecular fingerprints. Chem. Biol. Drug Des. 2008, 71, 8-14. [CrossRef]

13. Awale, M.; Reymond, J.L. The polypharmacology browser: A web-based multi-fingerprint target prediction tool using chembl bioactivity data. J. Cheminform. 2017, 9, 11. [CrossRef]

14. Geppert, H.; Bajorath, J. Advances in 2d fingerprint similarity searching. Expert Opin. Drug Discov. 2010, 5, 529-542. [CrossRef]

15. Muegge, I.; Mukherjee, P. An overview of molecular fingerprint similarity search in virtual screening. Expert Opin. Drug. Discov. 2016, 11, 137-148. [CrossRef]

16. O'Boyle, N.M.; Sayle, R.A. Comparing structural fingerprints using a literature-based similarity benchmark. J. Cheminform. 2016, 8, 36. [CrossRef]

17. Willett, P. Similarity searching using 2d structural fingerprints. Meth. Mol. Biol. 2011, 672, 133-158.

18. Durant, J.L.; Leland, B.A.; Henry, D.R.; Nourse, J.G. Reoptimization of mdl keys for use in drug discovery. J. Chem. Inf. Comput. Sci. 2002, 42, 1273-1280. [CrossRef]

19. Carhart, R.E.; Smith, D.H.; Venkataraghavan, R. Atom pairs as molecular-features in structure activity studies-Definition and applications. J. Chem. Inf. Comp. Sci. 1985, 25, 64-73. [CrossRef]

20. Nilakantan, R.; Bauman, N.; Dixon, J.S.; Venkataraghavan, R. Topological torsion-A new molecular descriptor for sar applications-Comparison with other descriptors. J. Chem. Inf. Comp. Sci. 1987, 27, 82-85. [CrossRef]

21. Rogers, D.; Hahn, M. Extended-connectivity fingerprints. J. Chem. Inf. Model. 2010, 50, 742-754. [CrossRef]

22. Hassan, M.; Brown, R.D.; Varma-O'brien, S.; Rogers, D. Cheminformatics analysis and learning in a data pipelining environment. Mol. Divers. 2006, 10, 283-299. [CrossRef]

23. Glen, R.C.; Bender, A.; Arnby, C.H.; Carlsson, L.; Boyer, S.; Smith, J. Circular fingerprints: Flexible molecular descriptors with applications from physical chemistry to adme. IDrugs 2006, 9, 199-204.

24. Riniker, S.; Landrum, G.A. Open-source platform to benchmark fingerprints for ligand-based virtual screening. J. Cheminform. 2013, 5, 26. [CrossRef]

25. O'Hagan, S.; Kell, D.B. Consensus rank orderings of molecular fingerprints illustrate the 'most genuine' similarities between marketed drugs and small endogenous human metabolites, but highlight exogenous natural products as the most important 'natural' drug transporter substrates. ADMET E DMPK 2017, 5, 85-125.

26. Dickens, D.; Rädisch, S.; Chiduza, G.N.; Giannoudis, A.; Cross, M.J.; Malik, H.; Schaeffeler, E.; Sison-Young, R.L.; Wilkinson, E.L.; Goldring, C.E.; et al. Cellular uptake of the atypical antipsychotic clozapine is a carrier-mediated process. Mol. Pharm. 2018, 15, 3557-3572. [CrossRef] [PubMed]

27. Weininger, D. SMILES, a chemical language and information system. 1. Introduction to methodology and encoding rules. J. Chem. Inf. Comput. Sci. 1988, 28, 31-36. [CrossRef]

28. Rumelhart, D.E.; McClelland, J.L. The PDP Research Group. Parallel Distributed Processing. Experiments in the Microstructure of Cognition; M.I.T. Press: Cambridge, MA, USA, 1986.

29. Goodacre, R.; Kell, D.B.; Bianchi, G. Rapid assessment of the adulteration of virgin olive oils by other seed oils using pyrolysis mass spectrometry and artificial neural networks. J. Sci. Food Agric. 1993, 63, $297-307$. [CrossRef]

30. Goodacre, R.; Timmins, É.M.; Burton, R.; Kaderbhai, N.; Woodward, A.M.; Kell, D.B.; Rooney, P.J. Rapid identification of urinary tract infection bacteria using hyperspectral whole-organism fingerprinting and artificial neural networks. Microbiology UK 1998, 144, 1157-1170. [CrossRef]

31. Tetko, I.V.; Gasteiger, J.; Todeschini, R.; Mauri, A.; Livingstone, D.; Ertl, P.; Palyulin, V.; Radchenko, E.; Zefirov, N.S.; Makarenko, A.S.; et al. Virtual computational chemistry laboratory-Design and description. J. Comput. Aided Mol. Des. 2005, 19, 453-463. [CrossRef]

32. O'Boyle, N.; Dalke, A. Deepsmiles: An Adaptation of Smiles for use in Machine-learning of Chemical Structures. ChemRxiv. 2018, 7097960.v7097961. Available online: https: //chemrxiv.org/articles/preprint/DeepSMILES_An_Adaptation_of_SMILES_for_Use_in_MachineLearning_of_Chemical_Structures/7097960 (accessed on 29 July 2020).

33. Segler, M.H.S.; Kogej, T.; Tyrchan, C.; Waller, M.P. Generating focussed molecule libraries for drug discovery with recurrent neural networks. ACS Central Sci. 2017, 4, 120-131. [CrossRef] 
34. Jin, W.; Barzilay, R.; Jaakkola, T. Junction Tree Variational Autoencoder for Molecular Graph Generation. arXiv 2018, arXiv:1802.04364v04362.

35. Kajino, H. Molecular Hypergraph Grammar with its Application to Molecular Optimization. arXiv 2018, arXiv:02745v02741.

36. Panteleev, J.; Gao, H.; Jia, L. Recent applications of machine learning in medicinal chemistry. Bioorg. Med. Chem. Lett. 2018, 28, 2807-2815. [CrossRef] [PubMed]

37. Jaeger, S.; Fulle, S.; Turk, S. Mol2vec: Unsupervised machine learning approach with chemical intuition. J. Chem. Inf. Model. 2018, 58, 27-35. [CrossRef]

38. Shibayama, S.; Marcou, G.; Horvath, D.; Baskin, I.I.; Funatsu, K.; Varnek, A. Application of the mol2vec technology to large-size data visualization and analysis. Mol. Inform. 2020, 39, e1900170. [CrossRef]

39. Duvenaud, D.; Maclaurin, D.; Aguilera-Iparraguirre, J.; Gómez-Bombarelli, R.; Hirzel, T.; Aspuru-Guzik, A.; Adams, R.P. Convolutional networks on graphs for learning molecular fingerprints. Adv. NIPS 2015, 2, 2224-2232.

40. Kearnes, S.; McCloskey, K.; Berndl, M.; Pande, V.; Riley, P. Molecular graph convolutions: Moving beyond fingerprints. J. Comput. Aided Mol. Des. 2016, 30, 595-608. [CrossRef]

41. Gupta, A.; Müller, A.T.; Huisman, B.J.H.; Fuchs, J.A.; Schneider, P.; Schneider, G. Generative recurrent networks for de novo drug design. Mol. Inform. 2018, 37, 1700111. [CrossRef]

42. Schneider, G. Generative models for artificially-intelligent molecular design. Mol. Inf. 2018, 37, 188031. [CrossRef]

43. Grisoni, F.; Schneider, G. De novo molecular design with generative long short-term memory. Chimia 2019, 73, 1006-1011. [CrossRef]

44. Arús-Pous, J.; Blaschke, T.; Ulander, S.; Reymond, J.L.; Chen, H.; Engkvist, O. Exploring the gdb-13 chemical space using deep generative models. J. Cheminform. 2019, 11, 20. [CrossRef]

45. Jørgensen, P.B.; Schmidt, M.N.; Winther, O. Deep generative models for molecular science. Mol. Inf. 2018, 37, 1700133. [CrossRef]

46. Li, Y.; Hu, J.; Wang, Y.; Zhou, J.; Zhang, L.; Liu, Z. Deepscaffold: A comprehensive tool for scaffold-based de novo drug discovery using deep learning. J. Chem. Inf. Model 2020, 60, 77-91. [CrossRef]

47. Lim, J.; Hwang, S.Y.; Moon, S.; Kim, S.; Kim, W.Y. Scaffold-based molecular design with a graph generative model. Chem. Sci. 2020, 11, 1153-1164. [CrossRef]

48. Moret, M.; Friedrich, L.; Grisoni, F.; Merk, D.; Schneider, G. Generative molecular design in low data regimes. Nat. Mach. Intell. 2020, 2, 171-180. [CrossRef]

49. van Deursen, R.; Ertl, P.; Tetko, I.V.; Godin, G. Gen: Highly efficient smiles explorer using autodidactic generative examination networks. J. Cheminform. 2020, 12, 22. [CrossRef]

50. Walters, W.P.; Murcko, M. Assessing the impact of generative ai on medicinal chemistry. Nat Biotechnol 2020, 38, 143-145. [CrossRef]

51. Yan, C.; Wang, S.; Yang, J.; Xu, T.; Huang, J. Re-balancing Variational Autoencoder Loss for Molecule Sequence Generation. arXiv 2019, arXiv:1910.00698v00691.

52. Winter, R.; Montanari, F.; Noé, F.; Clevert, D.A. Learning continuous and data-driven molecular descriptors by translating equivalent chemical representations. Chem. Sci. 2019, 10, 1692-1701. [CrossRef]

53. Samanta, B.; De, A.; Ganguly, N.; Gomez-Rodriguez, M. Designing Random Graph Models using Variational Autoencoders with Applications to Chemical Design. arXiv 2018, arXiv:1802.05283.

54. Krenn, M.; Häse, F.; Nigam, A.; Friederich, P.; Aspuru-Guzik, A. Self-Referencing Embedded Strings (selfies): A 100\% Robust Molecular String Representation. arXiv 2019, arXiv:1905.13741.

55. Sattarov, B.; Baskin, I.I.; Horvath, D.; Marcou, G.; Bjerrum, E.J.; Varnek, A. De novo molecular design by combining deep autoencoder recurrent neural networks with generative topographic mapping. J. Chem. Inf. Model. 2019, 59, 1182-1196. [CrossRef]

56. Bengio, Y.; Courville, A.; Vincent, P. Representation learning: A review and new perspectives. IEEE Trans. Patt. Anal. Mach. Intell. 2013, 35, 1798-1828. [CrossRef]

57. Bousquet, O.; Gelly, S.; Tolstikhin, I.; Simon-Gabriel, C.-J.; Schoelkopf, B. From Optimal Transport to Generative Modeling: The Vegan Cookbook. arXiv 2017, arXiv:1705.07642.

58. Husain, H.; Nock, R.; Williamson, R.C. Adversarial Networks and Autoencoders: The Primal-dual Relationship and Generalization Bounds. arXiv 2019, arXiv:1902.00985. 
59. Goodfellow, I.J.; Pouget-Abadie, J.; Mirza, M.; Xu, B.; Warde-Farley, D.; Ozairy, S.; Courville, A.; Bengio, Y. Generative adversarial nets. arXiv 2014, arXiv:1406.2661v1401.

60. Polykovskiy, D.; Zhebrak, A.; Vetrov, D.; Ivanenkov, Y.; Aladinskiy, V.; Mamoshina, P.; Bozdaganyan, M.; Aliper, A.; Zhavoronkov, A.; Kadurin, A. Entangled conditional adversarial autoencoder for de novo drug discovery. Mol. Pharm. 2018, 15, 4398-4405. [CrossRef]

61. Arjovsky, M.; Chintala, S.; Bottou, L. Wasserstein gan. arXiv 2017, arXiv:1701.07875v07873.

62. Goodfellow, I. Generative adversarial networks. arXiv 2017, arXiv:1701.00160v00161.

63. Foster, D. Generative Deep Learning; O’Reilly: Sebastopol, CA, USA, 2019.

64. Langr, J.; Bok, V. Gans in Action; Manning: Shelter Island, NY, USA, 2019.

65. Prykhodko, O.; Johansson, S.V.; Kotsias, P.C.; Arús-Pous, J.; Bjerrum, E.J.; Engkvist, O.; Chen, H.M. A de novo molecular generation method using latent vector based generative adversarial network. J. Cheminform. 2019, 11, 74. [CrossRef]

66. Zhao, J.J.; Kim, Y.; Zhang, K.; Rush, A.M.; LeCun, Y. Adversarially Regularized Autoencoders for Generating Discrete Structures. arXiv 2017, arXiv:1706.04223v04221.

67. Kingma, D.; Welling, M. Auto-encoding variational bayes. arXiv 2014, arXiv:1312.6114v1310.

68. Rezende, D.J.; Mohamed, S.; Wierstra, D. Stochastic Backpropagation and Approximate Inference in Deep Generative Models. arXiv 2014, arXiv:1401.4082v1403.

69. Doersch, C. Tutorial on Variational Autoencoders. arXiv 2016, arXiv:1606.05908v05902.

70. Benhenda, M. Chemgan Challenge for Drug Discovery: Can ai Reproduce Natural Chemical Diversity? arXiv 2017, arXiv:1708.08227v08223.

71. Griffiths, R.-R.; Hernández-Lobato, J.M. Constrained Bayesian Optimization for Automatic Chemical Design. arXiv 2017, arXiv:1709.05501v05505.

72. Aumentado-Armstrong, T. Latent Molecular Optimization for Targeted Therapeutic Design. arXiv 2018, arXiv:1809.02032.

73. Blaschke, T.; Olivecrona, M.; Engkvist, O.; Bajorath, J.; Chen, H.M. Application of generative autoencoder in de novo molecular design. Mol. Inform. 2018, 37, 1700123. [CrossRef]

74. Gómez-Bombarelli, R.; Wei, J.N.; Duvenaud, D.; Hernández-Lobato, J.M.; Sánchez-Lengeling, B.; Sheberla, D.; Aguilera-Iparraguirre, J.; Hirzel, T.D.; Adams, R.P.; Aspuru-Guzik, A. Automatic chemical design using a data-driven continuous representation of molecules. ACS Cent. Sci. 2018, 4, 268-276. [CrossRef]

75. Tschannen, M.; Bachem, O.; Lucic, M. Recent Advances in Autoencoder-based Representation Learning. arXiv 2018, arXiv:1812.05069v05061.

76. Kingma, D.P.; Welling, M. An Introduction to Variational Autoencoders. arXiv 2019, arXiv:1906.02691v02691.

77. Rezende, D.J.; Viola, F. Taming vaes. arXiv 2018, arXiv:1810.00597v00591.

78. Hutson, M. Core progress in ai has stalled in some fields. Science 2020, 368, 927. [CrossRef]

79. Burgess, C.P.; Higgins, I.; Pal, A.; Matthey, L.; Watters, N.; Desjardins, G.; Lerchner, A. Understanding disentangling in $\beta$-vae. arXiv 2018, arXiv:1804.03599.

80. Taghanaki, S.A.; Havaei, M.; Lamb, A.; Sanghi, A.; Danielyan, A.; Custis, T. Jigsaw-vae: Towards Balancing Features in Variational Autoencoders. arXiv 2020, arXiv:2005.05496.

81. Caterini, A.; Cornish, R.; Sejdinovic, D.; Doucet, A. Variational Inference with Continuously-Indexed Normalizing Flows. arXiv 2020, arXiv:2007.05426.

82. Nielsen, D.; Jaini, P.; Hoogeboom, E.; Winther, O.; Welling, M. Survae flows: Surjections to bridge the Gap between Vaes and Flows. arXiv 2020, arXiv:2007.02731.

83. Li, Y.; Yu, S.; Principe, J.C.; Li, X.; Wu, D. Pri-vae: Principle-of-relevant-information Variational Autoencoders. arXiv 2020, arXiv:2007.06503.

84. Wolpert, D.H.; Macready, W.G. No free lunch theorems for optimization. IEEE Trans. Evol. Comput. 1997, 1, 67-82. [CrossRef]

85. Vaswani, A.; Shazeer, N.; Parmar, N.; Uszkoreit, J.; Jones, L.; Gomez, A.N.; Kaiser, L.; Polosukhin, I. Attention is All You Need. arXiv 2017, arXiv:1706.03762.

86. Devlin, J.; Chang, M.-W.; Lee, K.; Toutanova, K. Bert: Pre-training of deep bidirectional transformers for language understanding. arXiv 2018, arXiv:1810.04805.

87. Dai, B.; Wipf, D. Diagnosing and Enhancing vae Models. arXiv 2019, arXiv:1903.05789v05782.

88. Asperti, A.; Trentin, M. Balancing Reconstruction Error and Kullback-leibler Divergence in Variational Autoencoders. arXiv 2020, arXiv:2002.07514v07511. 
89. Goodacre, R.; Pygall, J.; Kell, D.B. Plant seed classification using pyrolysis mass spectrometry with unsupervised learning: The application of auto-associative and kohonen artificial neural networks. Chemometr. Intell. Lab. Syst. 1996, 34, 69-83. [CrossRef]

90. Yao, X. Evolving artificial neural networks. Proc. IEEE 1999, 87, 1423-1447.

91. Floreano, D.; Dürr, P.; Mattiussi, C. Neuroevolution: From architectures to learning. Evol. Intell. 2008, 1, 47-62. [CrossRef]

92. Vassiliades, V.; Christodoulou, C. Toward nonlinear local reinforcement learning rules through neuroevolution. Neural Comput. 2013, 25, 3020-3043. [CrossRef]

93. Stanley, K.O.; Clune, J.; Lehman, J.; Miikkulainen, R. Designing neural networks through neuroevolution. Nat. Mach. Intell. 2019, 1, 24-35. [CrossRef]

94. Iba, H.; Noman, N. Deep Neural Evolution: Deep Learning with Evolutionary Computation; Springer: Berlin, Germany, 2020.

95. Le Cun, Y.; Denker, J.S.; Solla, S.A. Optimal brain damage. Adv. Neural Inf. Proc. Syst. 1990, 2, 598-605.

96. Dietterich, T.G. Ensemble methods in machine learning. LNCS 2000, 1857, 1-15.

97. Hinton, G.E.; Srivastava, N.; Krizhevsky, A.; Sutskever, I.; Salakhutdinov, R.R. Improving neural networks by preventing co-adaptation of feature detectors. arXiv 2012, arXiv:1207.0580.

98. Keskar, N.S.; Mudigere, D.; Nocedal, J.; Smelyanskiy, M.; Tang, P.T.P. On large-batch training for deep learning: Generalization gap and sharp minima. arXiv 2017, arXiv:1609.04836v04832.

99. O'Hagan, S.; Swainston, N.; Handl, J.; Kell, D.B. A 'rule of $0.5^{\prime}$ for the metabolite-likeness of approved pharmaceutical drugs. Metabolomics 2015, 11, 323-339. [CrossRef]

100. O'Hagan, S.; Kell, D.B. Understanding the foundations of the structural similarities between marketed drugs and endogenous human metabolites. Front. Pharmacol. 2015, 6, 105. [CrossRef]

101. O'Hagan, S.; Kell, D.B. Metmaxstruct: A tversky-similarity-based strategy for analysing the (sub)structural similarities of drugs and endogenous metabolites. Front. Pharmacol. 2016, 7, 266. [CrossRef] [PubMed]

102. O'Hagan, S.; Kell, D.B. Analysis of drug-endogenous human metabolite similarities in terms of their maximum common substructures. J. Cheminform. 2017, 9, 18. [CrossRef] [PubMed]

103. O'Hagan, S.; Kell, D.B. Analysing and navigating natural products space for generating small, diverse, but representative chemical libraries. Biotechnol. J. 2018, 13, 1700503. [CrossRef] [PubMed]

104. O'Hagan, S.; Kell, D.B. Structural Similarities between Some Common Fluorophores used in Biology and Marketed drugs, Endogenous Metabolites, and Natural Products. bioRxiv 2019, 834325. Available online: https://www.biorxiv.org/content/10.1101/834325v1.abstract (accessed on 29 July 2020).

105. Samanta, S.; O’Hagan, S.; Swainston, N.; Roberts, T.J.; Kell, D.B. Vae-sim: A novel Molecular Similarity Measure Based on a Variational Autoencoder. bioRxiv 2020, 172908. Available online: https://www.biorxiv. org/content/10.1101/2020.06.26.172908v1.abstract (accessed on 29 July 2020).

106. Dai, H.; Tian, Y.; Dai, B.; Skiena, S.; Song, L. Syntax-Directed Variational Autoencoder for Structured data. arXiv 2018, arXiv:1802.08786v08721.

107. Kusner, M.J.; Paige, B.; Hernández-Lobato, J.M. Grammar Variational Autoencoder. arXiv 2017, arXiv:1703.01925v01921.

108. Kingma, D.P.; Ba, J.L. Adam: A Method for Stochastic Optimization. arXiv 2015, arXiv:1412.6980v1418.

109. Glorot, X.; Bengio, Y. Understanding the difficulty of training deep feedforward neural networks. Proc. AISTATs 2010, 9, 249-256.

110. O'Hagan, S.; Kell, D.B. The knime workflow environment and its applications in genetic programming and machine learning. Genetic Progr. Evol. Mach. 2015, 16, 387-391. [CrossRef]

111. McInnes, L.; Healy, J.; Melville, J. Umap: Uniform Manifold Approximation and Projection for Dimension Reduction. arXiv 2018, arXiv:1802.03426v03422.

112. McInnes, L.; Healy, J.; Saul, N.; Großberger, L. Umap: Uniform manifold approximation and projection. J. Open Source Software 2018. [CrossRef]

113. Citraro, R.; Leo, A.; Aiello, R.; Pugliese, M.; Russo, E.; De Sarro, G. Comparative analysis of the treatment of chronic antipsychotic drugs on epileptic susceptibility in genetically epilepsy-prone rats. Neurotherapeutics 2015, 12, 250-262. [CrossRef]

114. Thorn, C.F.; Muller, D.J.; Altman, R.B.; Klein, T.E. Pharmgkb summary: Clozapine pathway, pharmacokinetics. Pharmacogenet. Genomics 2018, 28, 214-222. [CrossRef] 
115. Hopkins, A.L.; Mason, J.S.; Overington, J.P. Can we rationally design promiscuous drugs? Curr. Opin. Struct. Biol. 2006, 16, 127-136. [CrossRef]

116. Mestres, J.; Gregori-Puigjané, E.; Valverde, S.; Solé, R.V. The topology of drug-target interaction networks: Implicit dependence on drug properties and target families. Mol. Biosyst. 2009, 5, 1051-1057. [CrossRef]

117. Mestres, J.; Gregori-Puigjané, E. Conciliating binding efficiency and polypharmacology. Trends Pharmacol. Sci. 2009, 30, 470-474. [CrossRef] [PubMed]

118. Oprea, T.I.; Bauman, J.E.; Bologa, C.G.; Buranda, T.; Chigaev, A.; Edwards, B.S.; Jarvik, J.W.; Gresham, H.D.; Haynes, M.K.; Hjelle, B.; et al. Drug repurposing from an academic perspective. Drug Discov. Today Ther. Strateg. 2011, 8, 61-69. [CrossRef] [PubMed]

119. Dimova, D.; Hu, Y.; Bajorath, J. Matched molecular pair analysis of small molecule microarray data identifies promiscuity cliffs and reveals molecular origins of extreme compound promiscuity. J. Med. Chem. 2012, 55, 10220-10228.

120. Peters, J.U.; Hert, J.; Bissantz, C.; Hillebrecht, A.; Gerebtzoff, G.; Bendels, S.; Tillier, F.; Migeon, J.; Fischer, H.; Guba, W.; et al. Can we discover pharmacological promiscuity early in the drug discovery process? Drug Discov. Today 2012, 17, 325-335. [CrossRef]

121. Hu, Y.; Gupta-Ostermann, D.; Bajorath, J. Exploring compound promiscuity patterns and multi-target activity spaces. Comput. Struct. Biotechnol. J. 2014, 9, e201401003. [CrossRef] [PubMed]

122. Bajorath, J. Molecular similarity concepts for informatics applications. Methods Mol. Biol. 2017, 1526, $231-245$. [PubMed]

123. Eckert, H.; Bajorath, J. Molecular similarity analysis in virtual screening: Foundations, limitations and novel approaches. Drug Discov. Today 2007, 12, 225-233. [CrossRef]

124. Medina-Franco, J.L.; Maggiora, G.M. Molecular similarity analysis. In Chemoinformatics for Drug Discovery; Bajorath, J., Ed.; Wiley: Hoboken, NJ, USA, 2014; pp. 343-399.

125. Zhang, B.; Vogt, M.; Maggiora, G.M.; Bajorath, J. Comparison of bioactive chemical space networks generated using substructure- and fingerprint-based measures of molecular similarity. J. Comput. Aided Mol. Des. 2015, 29, 595-608. [CrossRef]

126. Hornik, K.; Stinchcombe, M.; White, H. Multilayer feedforward networks are universal approximators. Neural Netw. 1989, 2, 359-366. [CrossRef]

127. Hornik, K. Approximation capabilities of multilayer feedforward networks. Neural Netw. 1991, 4, $251-257$. [CrossRef]

128. Everitt, B.S. Cluster Analysis; Edward Arnold: London, UK, 1993.

129. Jain, A.K.; Dubes, R.C. Algorithms for Clustering Data; Prentice Hall: Englewood Cliffs, NJ, USA, 1988.

130. Kaufman, L.; Rousseeuw, P.J. Finding Groups in Data. An Introduction to Cluster Analysis; Wiley: New York, NY, USA, 1990.

131. Handl, J.; Knowles, J.; Kell, D.B. Computational cluster validation in post-genomic data analysis. Bioinformatics 2005, 21, 3201-3212. [CrossRef] [PubMed]

132. MacCuish, J.D.; MacCuish, N.E. Clustering in Bioinformatics And Drug Discovery; CRC Press: Boca Raton, FL, USA, 2011.

133. Hong, S.H.; Ryu, S.; Lim, J.; Kim, W.Y. Molecular generative model based on an adversarially regularized autoencoder. J. Chem. Inf. Model. 2020, 60, 29-36. [CrossRef] [PubMed]

134. Bozkurt, A.; Esmaeili, B.; Brooks, D.H.; Dy, J.G.; van de Meent, J.-W. Evaluating Combinatorial Generalization in Variational Autoencoders. arXiv 2019, arXiv:1911.04594v04591.

135. Bozkurt, A.; Esmaeili, B.; Brooks, D.H.; Dy, J.G.; van de Meent, J.-W. Can Vaes Generate novel Examples? arXiv 2018, arXiv:1812.09624v09621.

Sample Availability: Samples of the compounds are not available from the authors. 